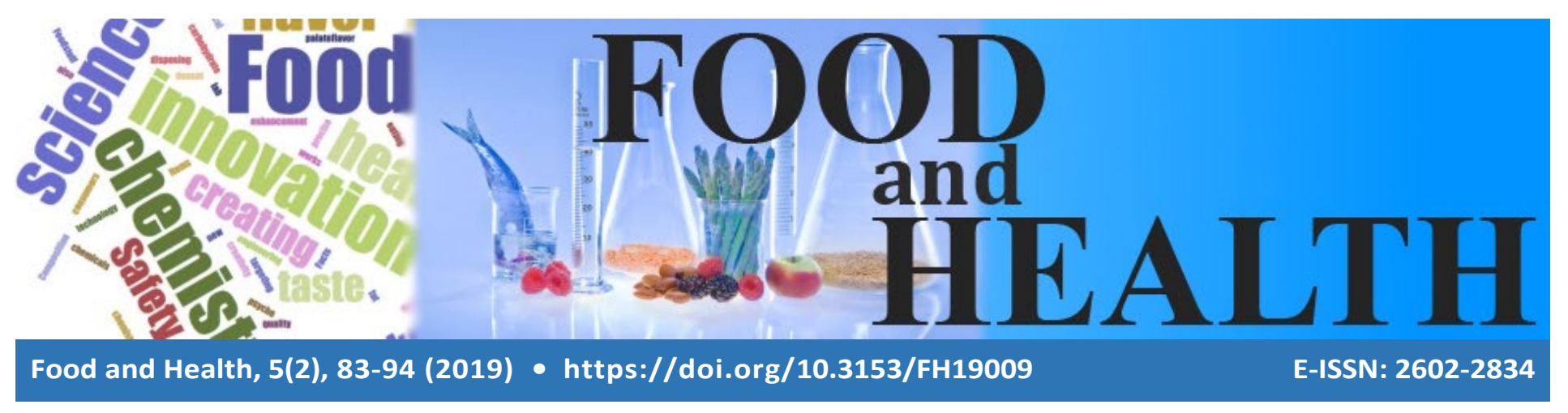

Research Article

\title{
THE ACQUISITION OF HEALTHY FOOD CHOICES, BREAKFAST AND EATING HABITS TO THE PRE-EDUCATION AND AFTER EDUCATION IN ADOLESCENTS
}

\author{
Aziz Aksoy ${ }^{\circledR}$, Gözde Ulaş (i)
}

Cite this article as:

Aksoy, A., Ulaş, G. (2019). The acquisition of healthy food choices, breakfast and eating habits to the pre-education and after education in adolecents. Food and Health, 5(2), 83-94. https://doi.org/10.3153/FH19009

Bitlis Eren University, School of Health, Department of Nutrition and Dietetics, Bitlis, Turkey

ORCID IDs of the authors: A.A. 0000-0002-9683-6691 G.U. 0000-0001-7221-513X

Submitted: 27.08 .2018

Accepted: 09.09.2018

Published online: 20.11.2018

Correspondence:

Aziz AKSOY

E-mail: aksoy aziz@hotmail.com

() Copyright 2019 by ScientificWebJournals Available online at http://jifhs.scientificwebjournals.com

\begin{abstract}
Adolescence is a period when the fastest growing happens in individuals after babyhood. The study was conducted with a total of 540 students from the $5^{\text {th }}, 6^{\text {th }}, 7^{\text {th }}$, and $8^{\text {th }}$ Grade students $(284 \mathrm{M}$ and 256F) attending secondary school. In the first stage, 540 questionnaires that had 39 questions were applied to the students who were trained; and the children were given nutrition training; and after 20 days, another 540 questionnaires were applied again. The data of a total of 540 pre-training and 540 post-training students were recorded in terms of their eating habits, regular breakfast and healthy food selection before and after the study. The body parameters according to gender (height, weight, BMI) show normality with the percentile values formed in Turkish children. The percentages of the answers given by the students to the questions before and after the training are given under each question category. A significant difference was detected between the habits of not having breakfast according to gender $(\mathrm{P}=0.021)(\mathrm{P}<0.05)$. As a conclusion, these data show that food selection may be developed with training.
\end{abstract}

Keywords: Adolescence, Nutrition training, Eating habits, BMI, Body parameters 


\section{Introduction}

Nutrition in adolescents is important for healthy growth and development. One of the periods in which sufficient and balanced nutrition is very important is the adolescence period, which is between childhood and maturity, where growth and development are accelerated. Adolescence is a period when the fastest growing happens in individuals after babyhood. Adolescence means growth and reaching maturity (Spear, 2002; Demirezen and Cosansu, 2005).

In this period, nutrition habits generally differ from children and adults. Adolescents have the tendency to skip meals and the tendency to eat most of the meals out of their homes, to consume fizzy drinks, candy, dietary foods and readymade meals and drinks is more (Stea et al., 2012). In the adolescence period, energy and nutrition items should be taken in adequate and balanced amounts; otherwise, the growth of the body may slow down, success at school may decrease; and important diseases such as cardiovascular, diabetes, hypertension may be seen in further ages (Malik et al., 2013).

There is no single nutrient element that contains all the necessary nutrients to meet the requirements of the human body (Aksoy and Selen, 2018). For this reason, the human body can only stay healthy if all nutrients are taken properly, balanced and correctly from all foods groups (Aksoy et al., 2017). Foods have been divided into different groups; and the amount of from food groups be consumed every day has been determined by national food guides over the world (Willett and McCullough, 2008; Aktas, 2011; TUBER, 2015).

Scientists in the food quides generally have grouped the nutrients under five groups (TUBER, 2015), the amounts of the nutrients included in these groups have been determined, and daily nutrition plans have been made. In this way, people have been guided to learn the calories and their varieties, which should be taken according to the needs of the body, the nutrient groups that are to be taken from outside when they consume nutrients (Cooke and Wardle, 2005; Neumark-Sztainer et al., 1999; Auestad et al., 2015).

If healthy eating habits, which will continue lifelong, are acquired during the adolescence period, possible future risks will be reduced. Regular breakfast habit is important for the health and development in adolescence and breakfast is as the most essential meal of the day, but it is the most skipped meal (Cebirbay et al, 2011). The energy and nutrients needed between 10-12 hours between dinner and breakfast are provided by the body storages. Breakfast time should not be missed after this time period. When breakfast is skipped, weakness, headache, attention and perception problems may appear during the day (Wouters et al., 2010).

In reinforcing healthy eating habits, which are acquired during childhood and adolescence, the school environment has a great importance. Schools offer many opportunities to promote healthy dietary and physical activity patterns for children, and are also preventing child malnutrition in all its forms (i.e. undernutrition, micronutrient deficiencies, and obesity and other nutrition related chronic diseases) (Aktas, 2017). Keeping foods like fruit, milk, and fruit-milk at school may be important in shaping the food variety and portion control at lunchtime. Non-alcoholic drinks that contain sugar that is consumed in schools and low-nutritional foods can cause that unhealthy eating habits to emerge (Wordell et al., 2012; Cebirbay et al., 2011). The present health conditions are important for adolescents; possible future health problems are not perceived as a worrying situation by them; and different priorities push health and nutrition issues into the background (Wouters et al., 2010).

In this study, the aim was to show how the training on nutrition at schools affect the level of nutrition habits of adolescents; and to determine the levels of the changes that were detected in the pre-training and post-training nutrition habits. In parallel to this; education in adolescents is aimed to show how effective they are in changes in eating habits

\section{Materials and Methods}

\section{Material}

The study was conducted with a total of 540 students from the $5^{\text {th }}, 6^{\text {th }}, 7^{\text {th }}$, and $8^{\text {th }}$ Grade students $(284$ males and 256 females) attending Alparslan Secondary School of Malazgirt district, National Education Directorate of Mus Turkey.

\section{Methods}

The study was conducted in two stages. In the first stage, 540 questionnaires that had 39 questions were applied to the students who were trained; and the children were given nutrition training; the training was taught by an expert dietician throughout a one-week, healthy and correct nutrition, healthy breakfast habits, healthy nutritional items, face and visual materials. Nutrition education provided by using modern education tools with the active participation of students. The training were given by the researchers. And after 20 days, another 540 questionnaires were applied again. The data of a total of 540 pre-training and 540 post-training students were recorded in terms of their eating habits, regular breakfast and healthy food selection before and after the 
training study. Training on nutrition habits had not been provided before in the study school. The experimental method was used in the study. The experimental design of the study is a single group pre-test, final-test model was applied in the study. The Questionnaire Model was used in the present study. The Questionnaire Form, which was developed as a data collection tool, was preferred because it is an appropriate tool in data collection. The questionnaire consisted of three parts. In the first part, there were questions on determining the information about the students and their families; in the second part, there were questions on determining the eating habits of the students; and in the third part, there were questions on determining the frequency of food consumption. The height measurements of the children were made by using the Harpenden Stadiometer (ADE/Hamburg, MZ10020) ultrasonic height measurement unit with an accuracy of $0.1 \mathrm{~cm}$ sensitivity, as the individual was standing parallel to the ground with nothing to affect the measurement, with light clothing; and shoes removed; the weight of the individuals were measured with the InBody230 (MW160) Bioelectrical Impedance Body Analyzer device. While the height and weight of the students were measured; body weight and height measurement procedures were applied to 12 and older age groups (TBSA, 2010). The data to determine the nutrient consumption frequency consisted of a 5-point Likert Scale as "Every day"; "5-6 times a week", "at least 3-4 times a week", "1-2 times a week", "Never". The Questionnaire Form was applied to the students who participated in the study under the surveillance of the author of the study. The necessary explanations about the questionnaire were made to the students; it was accepted that the students gave accurate and unbiased answers to the questions.

\section{Statistical Analysis}

The questionnaire data were entered into Microsoft Excel; and the statistical evaluation of the data was made with the IBM SSPS Statistics $17 \circledR$ Package Program; and the results were evaluated by performing T-test, ANOVA and correlation tests. A value of $\mathrm{P}<0.05$ was considered to be statistically significant.

\section{Ethical Principles}

The study protocol was approved by the Secretary General's Office of Bitlis Eren University on 22.12.2016 and with the Ethical Board Approval with the number E.3624 on 13.12.2016 with 2016/16-VIII decision.

\section{Results and Discussion}

\section{Demographical Characteristic}

No specific selections were made for the gender distributions of the students who were included in the present study. Participation was formed a class-based manner, and the age range was distributed according to the numbers of the students in the classes. The number of students who were 10 years old due to the early start to school, and the ones who were 10 years of age due to the late start to school was also included in the table.

Table 1. The gender, age and grade distributions of the students

\begin{tabular}{lccc}
\hline Gender distribution of students & & & \\
Gender & $\mathrm{n}$ & $\%$ & Total \\
\hline Boys (Male) & 284 & 52.6 & 540 \\
\hline Girls (Female) & 256 & 47.4 & 100.0 \\
\hline
\end{tabular}

\section{Distribution of students according to classes}

\begin{tabular}{|c|c|c|c|c|c|c|}
\hline Classes & $5^{\text {th }}$ Class & $6^{\text {th }}$ Class & $7^{\text {th }}$ Class & $8^{\text {th }}$ Class & \multicolumn{2}{|c|}{ Total } \\
\hline $\mathrm{n}$ & 151 & 122 & 138 & 129 & \multicolumn{2}{|c|}{540} \\
\hline$\%$ & 27.9 & 22.6 & 25.6 & 23.9 & \multicolumn{2}{|c|}{100.0} \\
\hline \multicolumn{7}{|c|}{ Body Mass Index (BMI) values by age groups comparisons } \\
\hline Age Range & $\mathrm{n}$ & Mean $\pm \mathrm{SD}$ & Minimum & Maximum & $F$ & $P$ \\
\hline 10 years old & 37 & $17.50 \pm 3.01$ & 12.81 & 25.57 & \multirow{7}{*}{5.497} & \multirow{7}{*}{0.000} \\
\hline 11 years old & 107 & $16.97 \pm 2.31$ & 10.06 & 22.26 & & \\
\hline 12 years old & 123 & $17.88 \pm 3.07$ & 13.13 & 26.84 & & \\
\hline 13 years old & 134 & $18.44 \pm 3.14$ & 11.11 & 32.47 & & \\
\hline 14 years old & 115 & $18.88 \pm 3.01$ & 12.50 & 29.14 & & \\
\hline 15 years old & 21 & $19.36 \pm 1.85$ & 16.98 & 23.15 & & \\
\hline 16 years old & 3 & $19.48 \pm 4.67$ & 14.87 & 24.22 & & \\
\hline
\end{tabular}

SD: Standard Deviation 
When the BMI values of the students were examined, they were ranked according to their ages and the BMI averages were given. The expected difference that was based on age was found to be significant $(\mathrm{P}<0.05)$.

When the educational status of the parents was examined it was determined that literacy levels in mothers were found to be $30.2 \%$ (163), in fathers $5.2 \%(28)$; again $3.3 \%$ (18) of the mothers and $14.4 \%$ (78) of the fathers were university graduates. There was a significant relation between fathers' educational levels and the having breakfast habits of the children $(\mathrm{P}=0.015)$; the income levels of the families and what the children brought to school to eat $(\mathrm{P}=0.021)$; and where the children ate lunch $(\mathrm{P}=0.028)(\mathrm{P}<0,05)$.

The average BMI, height and weight values based on gender and age of the students are given in Table 3.

Table 2. Educational status of the parents

\begin{tabular}{lllll}
\hline & \multicolumn{2}{c}{ Father education } & \multicolumn{2}{c}{ Mother education } \\
\hline & $\mathbf{n}$ & $\mathbf{\%}$ & $\mathbf{n}$ & $\mathbf{\%}$ \\
\hline Reader-Writer Not & 163 & 30.2 & 28 & 5.2 \\
\hline Primary education & 298 & 55.2 & 221 & 40.9 \\
\hline High school & 61 & 11.3 & 213 & 39.4 \\
\hline University & 18 & 3.3 & 78 & 14.4 \\
\hline Total & 540 & 100.0 & 540 & 100.0 \\
\hline
\end{tabular}

Table 3. Body measurement analysis values according to gender and age

\begin{tabular}{|c|c|c|c|c|c|c|}
\hline & \multicolumn{3}{|c|}{ Boys (M) } & \multicolumn{2}{|c|}{ Girls (F) } & \multirow[b]{2}{*}{$P$} \\
\hline & Age & $\mathbf{n}$ & Mean \pm SD & $\mathbf{n}$ & Mean \pm SD & \\
\hline \multirow{7}{*}{ BMI } & 10.00 & 20 & $17.37 \pm 2.61$ & 17 & $17.64 \pm 3.50$ & \multirow{7}{*}{0.043} \\
\hline & 11.00 & 59 & $17.56 \pm 2.23$ & 48 & $16.26 \pm 2.23$ & \\
\hline & 12.00 & 69 & $18.29 \pm 3.08$ & 54 & $17.37 \pm 3.00$ & \\
\hline & 13.00 & 64 & $18.71 \pm 3.29$ & 70 & $18.20 \pm 3.01$ & \\
\hline & 14.00 & 60 & $18.81 \pm 2.65$ & 55 & $18.96 \pm 3.38$ & \\
\hline & 15.00 & 11 & $19.40 \pm 2.05$ & 10 & $19.32 \pm 1.72$ & \\
\hline & 16.00 & 1 & 24.22 & 2 & $17.11 \pm 3.17$ & \\
\hline \multirow{7}{*}{ Height } & 10.00 & 20 & $1.37 \pm 0.05$ & 17 & $1.39 \pm 0.07$ & \multirow{7}{*}{0.664} \\
\hline & 11.00 & 59 & $1.40 \pm 0.09$ & 48 & $1.37 \pm 0.07$ & \\
\hline & 12.00 & 69 & $1.45 \pm 0.09$ & 54 & $1.46 \pm 0.09$ & \\
\hline & 13.00 & 64 & $1.52 \pm 0.07$ & 70 & $1.53 \pm 0.07$ & \\
\hline & 14.00 & 60 & $1.58 \pm 0.10$ & 55 & $1.56 \pm 0.08$ & \\
\hline & 15.00 & 11 & $1.62 \pm 0.10$ & 10 & $1.54 \pm 0.09$ & \\
\hline & 16.00 & 1 & 1.60 & 2 & $1.65 \pm 0.02$ & \\
\hline \multirow{7}{*}{ Weight } & 10.00 & 20 & $32.85 \pm 5.65$ & 17 & $34.11 \pm 1.67$ & \multirow{7}{*}{0.830} \\
\hline & 11.00 & 59 & $35.05 \pm 7.30$ & 48 & $30.87 \pm 6.17$ & \\
\hline & 12.00 & 69 & $38.84 \pm 7.19$ & 54 & $37.50 \pm 8.42$ & \\
\hline & 13.00 & 64 & $43.84 \pm 8.76$ & 70 & $42.91 \pm 7.12$ & \\
\hline & 14.00 & 60 & $47.55 \pm 9.17$ & 55 & $46.03 \pm 7.86$ & \\
\hline & 15.00 & 11 & $51.72 \pm 9.26$ & 10 & $46.20 \pm 6.81$ & \\
\hline & 16.00 & 1 & 62.00 & 2 & $47.00 \pm 9.89$ & \\
\hline
\end{tabular}


Table 4.The nutrition habits of the students before and after the training

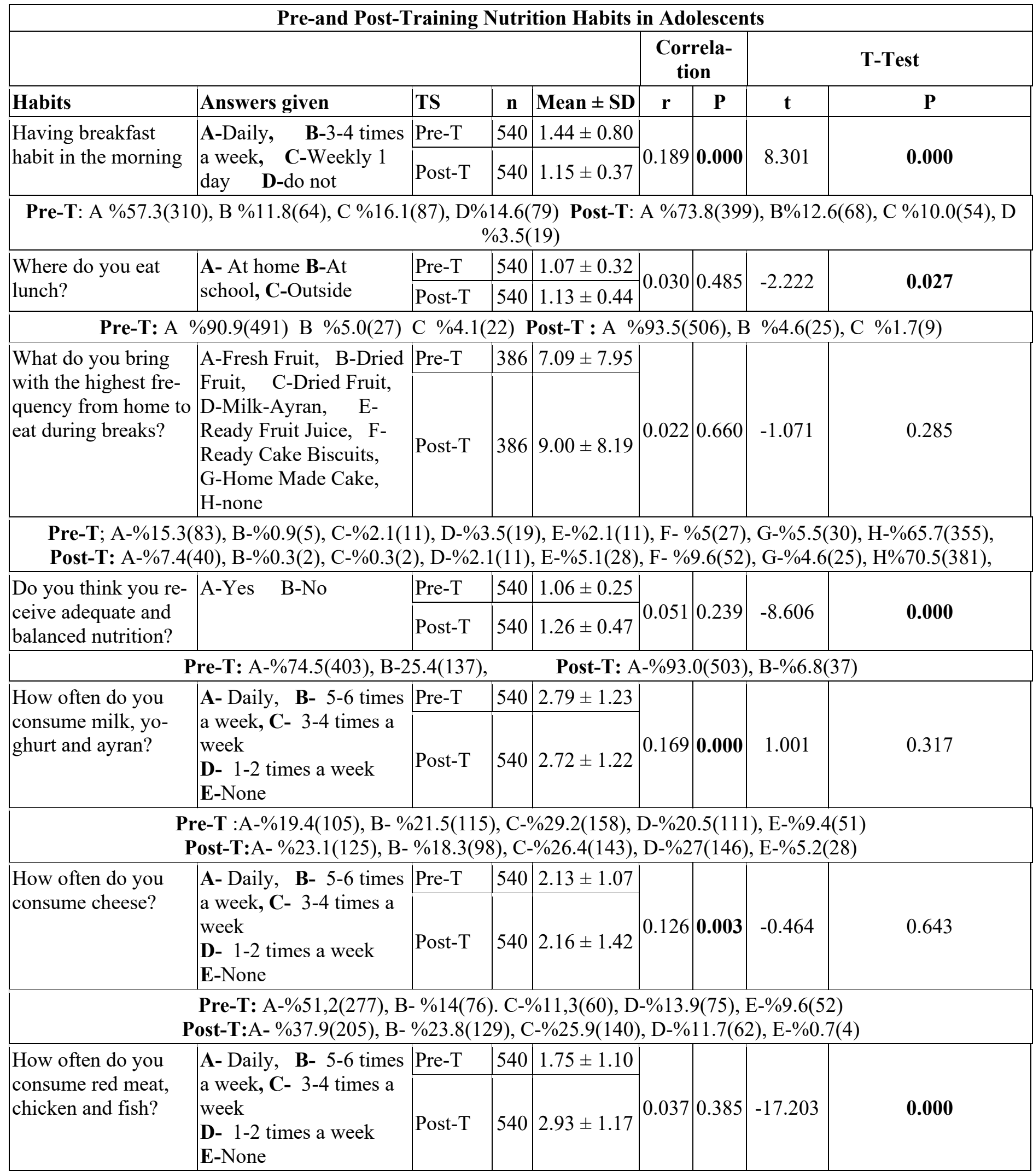


Pre-T: A-\%59.1(320), B- \%19(103). C-\%11,8(63). D-\%6.5(35), E-\%3.5(19)

Post-T:A- \%15.2(82), B- \%19.6(105), C-\%29.8(161), D-\%27.9(151), E-\%7.6(41)

How often do you consume egg?

\begin{tabular}{|l|l|l|l|l|l|l|l|} 
A- Daily, B- 5-6 times & Pre-T & 540 & $2.49 \pm 1.06$ & & & & \\
$\begin{array}{l}\text { a week, C- 3-4 times a } \\
\text { week } \\
\text { D- 1-2 times a week } \\
\text { E-None }\end{array}$ & Post-T & 540 & $2.92 \pm 1.29$ & 0.144 & $\mathbf{0 . 0 0 1}$ & -6.510 & \\
\hline
\end{tabular}

$\mathbf{0 . 0 0 0}$

Pre-T: A-\%21.1(114), B- \%29.4(159), C-\%30.7(166), D-\%16.5(88), E-\%2.4(13)

Post-T:A- \%20.7(112), B- \%15(81). C-\%24.6(132), D-\%30.1(163), E-\%9.6(52)

How often do you consume legumes?

\begin{tabular}{|l|l|l|l|l|l|l|} 
A- Daily, B- 5-6 times & Pre-T & 540 & $2.47 \pm 1.20$ & & & \\
$\begin{array}{l}\text { a week, C- 3-4 times a } \\
\text { week } \\
\text { D- 1-2 times a week } \\
\text { E-None }\end{array}$ & Post-T & 540 & $3.37 \pm 1.14$ & 0.226 & $\mathbf{0 . 0 0 0}$ & -14.187 \\
\hline
\end{tabular}

$\mathbf{0 . 0 0 0}$

Pre-T: A-\%29(157), B- \%21.5(115), C-\%26.6(144), D-\%18.7(101), E-\%4.3(23)

Post-T:A- \%10.4(56), B- \%9.8(53), C-\%24.8(134), D-\%42.3(228), E-\%12.8(69)

How often do you consume green-leaf vegetables?

\begin{tabular}{|c|c|c|c|c|c|c|}
\hline A- Daily, B- 5-6 times & Pre-T & 540 & $2.87 \pm 1.18$ & & & \\
\hline $\begin{array}{l}\text { week } \\
\text { D- } 1 \text {-2 times a week } \\
\text { E-None }\end{array}$ & Post-T & 540 & $3.17 \pm 1.24$ & 0.164 & 0.000 & -4.402 \\
\hline
\end{tabular}

Pre-T: A-\%16.3(88), B- \%21.4(116), C-\%26.8(144), D-\%29(157). E-\%6,5(35)

Post-T:A- \%12.8(69), B- \%17.6(94), C-\%23.7(128), D-\%31.4(170), E-\%14.6(79)

How often do you consume Potatoes?
\begin{tabular}{|l|l|l|l|l|} 
A- Daily, B- 5-6 times & Pre-T & 540 & $2.77 \pm 1.28$ \\
\hline
\end{tabular}
a week, C- 3-4 times a
week
D- 1-2 times a week
E-None
Pre-T: A-\%21.8(118), B- \%20.1(109), C-\%26.3(141), D-\%21.8(118), E-\%10(54)
Post-T:A- \%15.9(86), B- \%21.5(115), C-\%27(146), D-\%32.2(174), E-\%3.5(19)

\begin{tabular}{l|l|l|l|l|}
\hline How often do you & A- Daily, B- 5-6 times & Pre-T & 540 & $2.49 \pm 1.11$ \\
\cline { 2 - 5 } & & &
\end{tabular} consume fresh fruits? a week, C- 3-4 times a week

D- 1-2 times a week E-None

\begin{tabular}{|l|l|l|l|l|l|}
\hline Post-T & 540 & $2.27 \pm 1.16$ & 0.130 & $\mathbf{0 . 0 0 2}$ & 3.458 \\
\hline
\end{tabular}

0.001

Pre-T: A-\%24.4(132), B- \%24.5(132), C-\%29.9(161), D-\%19.4(105), E-\%1.8(10)

Post-T:A- \%35.3(191), B- \%22.4(120), C-\%24.4(132), D-\%15.5(84), E-\%2.4(13)

How often do you consume bread?
A- Daily, B- 5-6 times a week, C- 3-4 times a week

D- 1-2 times a week E-None

\begin{tabular}{|l|l|l|l|l|l|} 
Pre-T & 540 & $1.92 \pm 1.06$ & & & \\
\cline { 1 - 2 } Post-T & 540 & $1.47 \pm 0.97$ & & & \\
\end{tabular}

Pre-T: A-\%47.5(257), B- \%23.7(127), C-\%18.3(99), D-\%9.6(52), E-\%0.9(5)

Post-T:A- \%76.5(414), B- \%8.7(47), C-\%7.6(41), D-\%5(27), E-\%2(11) 


\begin{tabular}{|l|l|l|l|l|l|l|l|}
\hline $\begin{array}{l}\text { How often do you } \\
\text { consume bulgur, rice } \\
\text { and pasta? }\end{array}$ & $\begin{array}{l}\text { A-Daily, B- 5-6 times } \\
\text { a week, C- 3-4 times a } \\
\text { week } \\
\text { D- 1-2 times a week } \\
\text { E-None }\end{array}$ & Post-T & 540 & $2.59 \pm 1.13$ & & & \\
\hline
\end{tabular}

Pre-T: A-\%76.9(415), B- \%9.4(51), C-\%8.7(47), D-\%3.7(20), E-\%1.3(7)

Post-T:A- \%22.2(120), B- \%22.8(123), C-\%31.6(171), D-\%20.2(109), E-\%3.1(17)

How often do you consume olives?
\begin{tabular}{|l|l|l|l|} 
A- Daily, B- 5-6 times & Pre-T & 540 & $2.17 \pm 1.13$ \\
\hline
\end{tabular} a week, C- 3-4 times a week
D- 1-2 times a week
E-None

\begin{tabular}{|l|l|l|l|l|l|}
\hline Post-T & 540 & $2.63 \pm 1.57$ & 0.102 & $\mathbf{0 . 0 1 8}$ & -5.888 \\
& & & & \\
\hline
\end{tabular}
88
Pre-T: A-\%37.8(204), B- \%24.1(130), C-\%23.7(128), D-\%12.2(66), E-\%2.2(12)
Post-T:A- \%38.7(209), B- \%12.8(69), C-\%14(76), D-\%15(81), E-\%19.4(105)

$\mathbf{0 . 0 0 0}$

How often do you consume butter? \begin{tabular}{|l|l|l|l|} 
A- Daily, B- 5-6 times & Pre-T & 540 & $2.66 \pm 1.55$ \\
\hline
\end{tabular} a week, C- 3-4 times a week

D- 1-2 times a week E-None \begin{tabular}{l|l|l|l|l|l} 
Post-T & 540 & $3.25 \pm 1.54$ & 0.098 & $\mathbf{0 . 0 2 2}$ & -6.536
\end{tabular}

0.000

Pre-T: A-\%36.3(196), B- \%14.6(79), C-\%14.4(78), D-\%15.3(83), E-\%19.2(104)

Post-T:A- \%22.6(122), B- \%10.7(58), C-\%16.6(90), D-\%18.9(102), E-\%31.1(168)

\begin{tabular}{|c|c|c|c|c|c|c|c|c|}
\hline \multirow{2}{*}{$\begin{array}{l}\text { How often do you } \\
\text { consume margarine? }\end{array}$} & \multirow{2}{*}{$\begin{array}{l}\text { A- Daily, B- 5-6 times } \\
\text { a week, C- 3-4 times a } \\
\text { week } \\
\text { D- 1-2 times a week } \\
\text { E-None }\end{array}$} & Pre-T & 540 & $3.77 \pm 1.31$ & \multirow[b]{2}{*}{0.169} & \multirow[b]{2}{*}{0.000} & & \multirow[b]{2}{*}{0.692} \\
\hline & & Post-T & 540 & $3.80 \pm 1.37$ & & & -0.397 & \\
\hline
\end{tabular}

Pre-T: A-\%10.6(57), B- \%7.4(40), C-\%14.1(76), D-\%29.6(160), E-\%38.3(207)

Post-T :A- \%11.9(64), B- \%6.9(37), C-\%14.6(79), D-\%22(119), E-\%44.6(241)

\begin{tabular}{|l|l|l|l|l|}
\hline What is your fre- & A- Daily, B- 5-6 times & Pre-T & 540 & $4.10 \pm 1.17$ \\
\hline
\end{tabular} quency of consuming a week, C- 3-4 times a drinks with sugar?

week

D- 1-2 times a week E-None

Pre-T: A-\%6.7(36), B- \%4.1(22), C-\%11.6(63), D-\%27.7(150), E-\%49.7(269)

Post-T:A- \%39.4(213), B- \%11.3(61), C-\%13.7(74), D-\%21.9(118), E-\%13.7(74)

How often do you consume jam and cream chocolate?

\begin{tabular}{|l|l|l|l|l|l|l|} 
A- Daily, B- 5-6 times & Pre-T & 540 & $3.05 \pm 1.50$ & & & \\
$\begin{array}{l}\text { a week, C- 3-4 times a } \\
\text { week } \\
\text { D- 1-2 times a week } \\
\text { E-None }\end{array}$ & Post-T & 540 & $2.75 \pm 1.48$ & 0.104 & $\mathbf{0 . 0 1 5}$ & 3.439 \\
\hline
\end{tabular}

Pre-T: A-\%25.6(138), B- \%12(65), C-\%16.3(88), D-\%23.7(128), E-\%22.4(121)

Post-T : A- \%31.3(269), B- \%15(81), C-\%16.3(88), D-\%21.5(116), E-\%15.9(86)

\section{How often do you} consume honey, boiled grape juice, or week sesame oil with boiled grape juice? \begin{tabular}{|l|l|l|l|} 
A- Daily, B- 5-6 times & Pre-T & 540 & $2.80 \pm 1.42$ \\
\hline
\end{tabular} a week, C- 3-4 times a

D- 1-2 times a week E-None \begin{tabular}{|l|l|l|l|l|l|} 
Post-T & 540 & $3.07 \pm 1.55$ & 0.212 & $\mathbf{0 . 0 0 0}$ & -3.377
\end{tabular}
0.001 
Pre-T: A-\%28(151), B- \%15.4(83), C-\%19.6(106), D-\%22.8(123), E-\%14.3(77)

Post-T:A- \%26.3(142), B- \%12.6(68), C-\%14.3(77), D-\%21.3(115), E-\%26.6(138)

How often do you consume desserts

\begin{tabular}{|l|l|l|l|} 
A- Daily, B- 5-6 times & Pre-T & 540 & $3.13 \pm 1.48$ \\
\hline
\end{tabular}

with milk and syrup? week

D- 1-2 times a week

E-None

\begin{tabular}{|l|l|l|l|l|l|}
\hline Post- $\mathrm{T}$ & 540 & $3.48 \pm 1.19$ & 0.131 & $\mathbf{0 . 0 0 2}$ & -4.478 \\
\hline
\end{tabular}

0.000

Pre-T: A-\%20.4(110), B- \%17(92), C-\%16.3(88), D-\%20.9(113), E-\%25.4(137)

Post-T:A- \%9.1(49), B- \%12(65), C-\%20.2(109), D-\%39.1(211), E-\%19.6(106)

How often do you consume coke, fizzy drinks, ready-made fruit juice?
A- Daily, B- 5-6 times

a week, C- 3-4 times a week

D- 1-2 times a week

E-None

\begin{tabular}{|l|l|l|l|l|}
\hline Pre-T & 540 & $3.69 \pm 1.11$ & & \\
\cline { 1 - 2 } Post-T & 540 & $3.23 \pm 1.38$ & 0.056 & 0.192 \\
\end{tabular}

6.171

$\mathbf{0 . 0 0 0}$

Pre-T: A-\%6.3(34), B- \%8.1(44), C-\%19.6(106), D-\%41.5(224), E-\%24.4(132)

Post-T:A- \%18(97), B- \%12.8(69), C-\%17(92), D-\%32(173), E-\%20.2(109)

How often do you consume tea?

\begin{tabular}{|l|l|l|l|l|l|l|} 
A- Daily, B- 5-6 times & Pre-T & 540 & $3.67 \pm 1.21$ & & & \\
$\begin{array}{l}\text { a week, C- 3-4 times a } \\
\text { week } \\
\text { D- 1-2 times a week } \\
\text { E-None }\end{array}$ & Post-T & 540 & $1.83 \pm 1.26$ & 0.001 & 0.981 & 24.461 \\
\hline
\end{tabular}

\section{$\mathbf{0 . 0 0 0}$}

Pre-T: A-\%63(340), B- \%12(65), C-\%9.3(50), D-\%10.2(55), E-\%5.6(30)

Post-T:A- \%56.3(304), B- \%16.5(89), C-\%11.3(61), D-\%10.6(57), E-\%5.4(29)

How often do you consume chips, biscuits, and cereals?

\begin{tabular}{|l|l|l|l|l|l|l|} 
A- Daily, B- 5-6 times & Pre-T & 540 & $1.92 \pm 1.25$ & & & \\
$\begin{array}{l}\text { a week, C- 3-4 times a } \\
\text { week } \\
\text { D- 1-2 times a week } \\
\text { E-None }\end{array}$ & Post-T & 540 & $2.96 \pm 1.36$ & 0.014 & 0.753 & -12.946 \\
\hline
\end{tabular}

\subsection{0}

Pre-T: A-\%18(97), B- \%12.8(69), C-\%17(92), D-\%32(173), E-\%20.3(109)

Post-T:A- \%19.8(107), B- \%18.3(99), C-\%24.8(134), D-\%19.8(107), E-\%17.2(93)

How often do you consume salami and sausages?

\begin{tabular}{|l|l|l|l|l|l|l|} 
A- Daily, B- 5-6 times & Pre-T & 540 & $3.49 \pm 1.23$ & & & \\
\cline { 2 - 4 } $\begin{array}{l}\text { a week, C- 3-4 times a } \\
\text { week } \\
\text { D- 1-2 times a week } \\
\text { E-None }\end{array}$ & Post-T & 540 & $3.48 \pm 1.36$ & 0.160 & $\mathbf{0 . 0 0 0}$ & 0.153 \\
\hline
\end{tabular}

\subsection{8}

Pre-T: A-\%9.1(49), B- \%11.5(62), C-\%25(135), D-\%30(162), E-\%24.4(132)

Post-T: A- \%11.3(61), B- \%15.7(85), C-\%17.8(96), D-\%23.9(129), E-\%31.3(169)

How often do you consume fermented sausages?

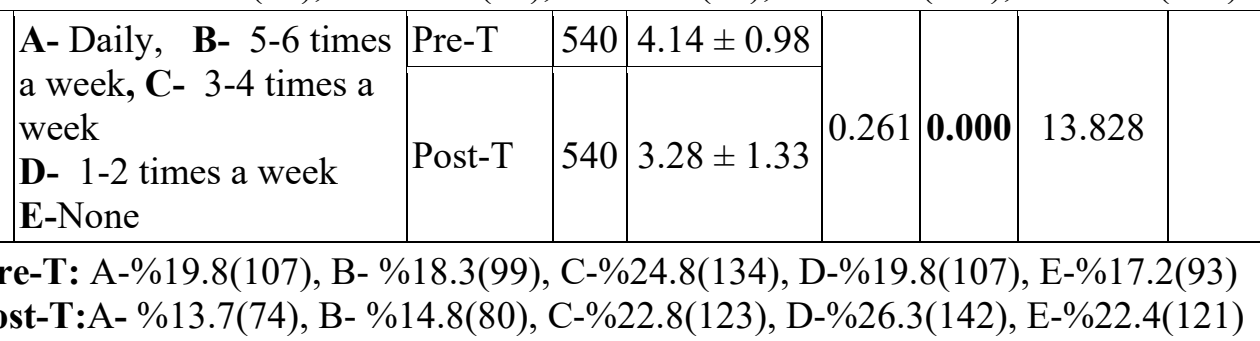

TS: Training Status; Pre-T: Pre-Training; Post-T: Post-Training; SD: Standard Deviation 
When the BMI values of the boys were compared with those of the girls according to age, the difference between them were found to be significant $(\mathrm{P}=0.043)$. No significant differences were detected between the height and weight values according to the gender variable $(\mathrm{P}>0.05)$. When the percentile values were compared in children according to body weights, it was determined that the weights of the males were between $25-50 \%$ percentile; height values were between 10-25 percentile; and the percentile values of the BMI averages were $25 \%$. It was also determined that the weights of the girls were between $25-50 \%$ percentile; their heights were between 10-25\% percentile; and the percentile value of the BMI average value was $15-25 \%$.

To perform an obesity evaluation in children and adolescents, the age and gender-specific BMI values were established. According to these evaluation criteria, BMIpercentile the value being below 5 shows malnutrition; its being between 5-85 shows normal weight; being between 86-95 shows being overweight (slightly obese), being over 95 shows obesity (Bundak et al., 2006; Neyzi et al., 1978; Neyzi et al., 2008). The body parameters according to gender (height, weight, BMI) show normality with the percentile values formed in Turkish children.

The nutrition habits of the students before and after the training are given in Table 4.

The percentages of the answers given by the students to the questions before and after the training are given under each question category. Significant differences were determined before and after the training between having breakfast in the morning $(\mathrm{r}=0.186)$; What is brought from home to eat during breaks $(\mathrm{r}=0.022)$; Thinking that $\mathrm{s} / \mathrm{he}$ has adequate and balanced nutrition $(\mathrm{r}=0.051)$; Consuming habits of milk, yogurt and ayran ( $\mathrm{r}=0.169)$; Red meat, chicken and fish $(\mathrm{r}=0.037)$; Eggs $(\mathrm{r}=0.144)$; Legumes $(\mathrm{r}=0.226)$; Green-leaf vegetables $(\mathrm{r}=0.164)$; Fresh fruit $(\mathrm{r}=0.130)$; Bread $(\mathrm{r}=0.085)$; Bulgur, rice and pasta $(\mathrm{r}=0.035)$; Olives $(\mathrm{r}=0.102)$; Butter $(\mathrm{r}=0.098)$; Drinks with sugar $(\mathrm{r}=0.103)$; Jam, cream chocolate $(\mathrm{r}=0.104)$; Honey, boiled grape juice or boiled grape juice with sesame oil ( $\mathrm{r}=0.212)$; Dessert with milk and syrup $(\mathrm{r}=0.131)$; Coke, fizzy drinks, ready-made fruit juice $(\mathrm{r}=0.056)$; Tea $(\mathrm{r}=-0.001)$; Chips, biscuits, cereals $(\mathrm{r}=-0.014)$ and Sausage $(\mathrm{r}=0.261)(\mathrm{P}<0.05)$; and a very weak a correlation was detected between them. There was a very weak and negative relationship between consumption habits of tea, chips, biscuit, and cereals. No significant differences were determined between the variables Where lunch is eaten $(\mathrm{r}=0,030)$; consuming Cheese $(\mathrm{r}=0.126)$; Potatoes $(\mathrm{r}=0.164)$; Margarine $(\mathrm{r}=0.169)$; Salami and sausage $(\mathrm{r}=0.160)$ before and after the training $(\mathrm{P}>0.05)$; however, a very weak and positive correlation was determined between them.

There are reports that children in school age are more likely to change their ways of doing than their right behavior. It is very common in children and adolescents to skip breakfast. It was determined that skipping breakfast was more in adolescent girls than in males (Steyn, 2010). This a situation has been explained with by fact that skipping breakfast was a weight control method in adolescent girls (Keshi-Rahkonen et al., 2003). Pulp, vitamins A, C and E, $\mathrm{B}_{6}, \mathrm{~B}_{12}$ and folate, iron, calcium, phosphorus, magnesium and potassium values of the individuals who skipped breakfast were found to be lower than in those who did not skip breakfast (Deshmukh-Taskar et al., 2010). In another study, it was reported that skipping breakfast could affect concentration, learning, and school performance (Story and Stang, 2005). In our study, a significant difference was detected between the habits of not having breakfast according to gender $(\mathrm{P}=0.021)(\mathrm{P}<0.05)$. Again, according to gender, there was a significant difference between healthy and balanced nutrition habits $(\mathrm{P}=0.001)(\mathrm{P}<0.05)$. It was found in a study conducted in the Mardin that overweight children between the ages of 7-15 consumed toast, coke, chips and chocolate more (Battaloglu, 2014); and in another study, it was reported those children ate bagels, chocolate, biscuits at the highest level, and preferred drinks like water, cola, fizzy drinks and fruit juice (Orhan and Celik, 2014). In our study, the rate of those who consumed coke, fizzy drinks and ready-made drinks 3-4 times a week was found to be $41.5 \%$ before the training; however, after the training, this rate decreased significantly. As a result of the training, when the benefits of other foods were learned, the diversity in food consumption increased; and after the training, the students consuming meat, poultry and fish showed a significant difference $(\mathrm{P}<0.05)$; however, a weak correlation $(\mathrm{r}=0.037)$ was also found between them. No significant differences were detected in terms of the gender variable $(\mathrm{P}>0.05)$. In another the study, a significant difference was reported based on the gender variable (Cebirbay et al., 2011). In a study conducted with children aged between 4 and 14 years and adolescents, it was determined that girls loved fruits and vegetables more than boys; and boys loved fatty and foods with sugar, meat products, processed meats and eggs more than girls (Cooke et al., 2005). In our study, a significant difference was detected after the training in the egg consumption of the students who consumed eggs every day before the training $(\mathrm{P}<0.05)$; and the relation between them was found to be very weak. According to the results of the research conducted before and after the training, sausages, tea, milk, fresh fruits and egg consumption (Sabbag and 
Surucuoglu, 2012). The relationship between pre-training and post-training data in our study was significant $(\mathrm{P}<0.05)$. In another a study, it was determined that most of the students who had very good, moderate and low nutrition knowledge drank tea; drinking coffee was very common among the subjects $(60.2 \%)$; and $26.1 \%$ of those who had very good nutrition knowledge consumed milk and ayran; and $20 \%$ of them consumed fruit juice (Birer and Ersoy, 1987). In our study, although a significant difference was detected in terms of consuming tea before and after the training, there was a very weak negative relationship between them. In a study that was conducted for the purpose of determining the nutrition knowledge and habits of senior high school students, it was determined that the students preferred coke at the intermediary meals were cola with $27.9 \%$; and the lowest consumed ones were yogurt and milk with $14.9 \%$ (Sagun, 1987). No significant differences were detected between the consumption frequencies for this product group $(\mathrm{P}>0.05)$.

In a study, it was determined in the weight and height measurements in boys and girls aged 12 years, girls were found to be overweight and taller than their boys (Neyzi et al., 1978). In the present study of ours, when we examined the 12-year age range participants, we found that their heights of the males and females were nearly the same; and there was a slight difference in the weight averages in favor of the males; however, the difference between males and females was not found to be significant $(\mathrm{P}>0.05)$. In another study, it was found that the sugar-chocolate consumption of the students was found to be $88 \%$ as once or twice or more per week; chips-potato consumption was like $71.7 \%$; and coke consumption were as $40.4 \%$ (Kutlu and Civi, 2009). In another a study, it was shown that the children consumed sweets and chocolate $(32.8 \%)$, chips, cracker etc. (15.4\%) and fizzy drinks (59\%) (Rakicioglu et al., 2000). In our study, after the training, those who said that they consumed coke and fizzy drinks 1-2 times a week were found to be $32 \%$, and those who said they consumed chips-biscuit-cereals were found to be $19.8 \%$. In a survey, results revealed $83.3 \%$ change in students' nutrition habits following education program (Sebbag, 2017). In our study this rate was found as $76.92 \%$.

\section{Conclusions}

As a conclusion, these data show that food selection may be developed with learning. As a result of the nutrition training provided by the dietitian in the adolescent children, there were significant differences despite the positive relation compared to the pre-training period. This study showed that it is important to provide nutrition trainings at schools in order to acquire healthy eating habits. Classes on healthy food selection and nutrition should be included in the curricula of schools and appropriate awareness about healthy lifestyle and its effects must be provided. The trainings should focus on healthy eating habits and lifestyles and should be given to all children in early staged of their developments.

\section{Compliance with Ethical Standard}

Conflict of interests: The authors declare that for this article they have no actual, potential or perceived the conflict of interests.

Ethics committee approval: The study protocol was approved by the Secretary General's Office of Bitlis Eren University on 22.12.2016 and with the Ethical Board Approval with the number E.3624 on 13.12.2016 with 2016/16-VIII decision.

Acknowledgment: Due to contributions; Mus province, Malazgirt district, Alparslan Secondary School, we thank the teachers and administrators

\section{References}

Aksoy, A., Selen, H. (2018). The evaluation of body composition and anthropometric measurements of males aged 18-25 years, based on the regularity of physical exercise. Progress in Nutrition, 20(3), 338-343.

Aksoy, A., Selen, H., Ozdemir, F.A., Bulut Arıkan, F. (2017). Association of physical activity and obesity status for individuals between the ages of 18-30 via bioelectrical impedance analysis device and metabolic holter measurements. Progress in Nutrition, 19(4), 391-397.

Aktas, N. (2011). Besin rehberleri: Beslenme eğitiminde gorsel bir arac. Selcuk Universitesi Sosyal Bilimler Enstitusu Dergisi, 25, 11-16.

Aktas, N. (2017). School-based nutrition promotion: nutrition friendly school program in Turkey. Mariateresa Gammone, Mehmet Ali Icbay, Hasan Arslan (Eds.), Recent Developments in Education (p. 479-486). International Association of Social Science Research. (Ebook), ISBN 978-83-943963-3-6.

Auestad, N., Hurley, J.S., Fulgoni, V.L., Schweitzer, C.M. (2015) contribution of food groups to energy and nutrient intakes in five developed countries. Nutrients, 7 , 4593-4618.

Battaloglu-Inanc, B. (2014). Okul çocuklarında doktor tanılı astım ve allerjik hastalıklarla fast-food ilişkisi. Konuralp Tip Dergisi, 6(2), 19-24. 
Birer, S. ve Ersoy, G. (1987). Metropoliten bir kentte spor yapan ve yapmayan üniversiteli k1z ögrencilerin beslenme bilgi ve alışkanlıkları. Beslenme ve Diyet Dergisi, 16(2), 153-170.

Bundak, R., Furman, A., Gunoz, H., Darendeliler, F., Bas, F., Neyzi, O. (2006). Body mass index references for Turkish children. Acta Paediatrica. 95, 194-198.

Cebirbay, M.A., Aktas, N., Calderoni, M. (2011). Determination of breakfast habits and knowledge of foreign undergraduates studying at Selcuk University in Turkey. Progress in Nutrition, 13(4), 276-285.

Cooke, L.J., Wardle, J. (2005). Age and gender differences in children's food preferences. British Journal of $\mathrm{Nu}$ trition, 93, 741-6.

Demirezen, E., Cosansu, G. (2005). Adolesan çağı öğrencilerde beslenme alışkanlıklarının degerlendirilmesi. Surekli Tip Egitimi Dergisi, 8, 174-178.

Deshmukh-Taskar, P.R., Nicklas, T.A., O’Neil, C.E., Keast, D.R., Radcliffe, J.D., Cho, S. (2010). The relationship of breakfast skipping and type of breakfast consumption with nutrient intake and weight status in children and adolescents: the National Health and Nutrition Examination Survey 1999-2006. Journal of the American Dietetic Association, 110(6), 869-878.

Keski-Rahkonen, A., Kaprio, J., Rissanen, A., Virkkunen, M., Rose, R.J. (2003). Breakfast skipping and healthcompromising behaviors in adolescents and adults. European Journal of Clinical Nutrition, 57(7), 842-853.

Kutlu, R., Civi, S. (2009). Özel bir ilköğretim okulu öğrencilerinde beslenme alışkanlıklarının ve beden kitle indekslerinin degerlendirilmesi, Firat Tip Dergisi, 14(1), $18-24$.

Malik, V.S., Pan, A., Willett, W.C., Hu, F.B. (2013). Sugarsweetened beverages and weight gain in children and adults: a systematic review and meta-analysis. American Journal of Clinical Nutritionü, 98, 1084-1102.

Neumark-Sztainer, D., Story, M., Perry, C., Casey, M.A. (1999). Factors influencing food choices of adolescents: findings from focus-group discussions with adolescents. Journal of the American Dietetic Association, 99, 929-937.
Neyzi, O., Binyildiz, P., Alp, H. (1978). Türk çocuklarında büyüme gelişme normları. İstanbul Tip Fakultesi Dergisi, 41(Supp 74), 3-22.

Neyzi, O., Gunoz, H., Furman, A., Bundak, R., Gokcay, G., Darendeliler, F., Bas, F. (2008). Turk çocuklarında vucut ağırlığı, boy uzunlugu, baş çevresi ve vücut kitle indeksi referans değerleri. Çocuk Sağlı̆̆i ve Hastalıkları Dergisi, 51, 1-14.

Orhan, A. Celik, İ. (2014). İlkögretim okulu öğrencilerinin kahvaltı alışkanlıkları ve okul kantini ile ilişkilerinin belirlenmesi, Akademik Gıda, 12(1), 51-56.

Rakicioglu, N., Karabudak, E., Kazanc, M., Yucecan, S. (2000). 10-18 Yaş grubu çocukların besin tüketim düzeyleri ve beslenme alışkanlıklarının saptanmasına yönelik bir çalışma. Uluslararası Beslenme ve Diyetetik Kongre Kitabl, C200, 156-157.

Sabbag C., Surucuoglu M.S. (2012). Influence of education on primary school students nutrition in Ankara. HealthMED, 6(2), 616-620.

Sabbag, C. (2017). The evaluation of short-term nutrition education on the nutrition habits of university students. International Journal of Human Sciences, 14(4), 36343644.

Sagun, P. (1987). Farklı sosyo-kültürel çevrelerdeki lise son sinif ögrencilerinin beslenme bilgi ve alışkanlıklarının ölçülmesi. Bilim Uzmanlığı Tezi. Hacettepe Üniversitesi, Ankara.

Spear, B.A. (2002). Adolescent growth and development. Journal of the American Dietetic Association, 102, 2329.

Stea, T.H., Overby, N.C., Klepp, K.I., Bere, E. (2012). Changes in beverage consumption in Norwegian children from 2001 to 2008. Public Health Nutrition, 15, 379-385.

Steyn, N. (2010). Does dietary knowledge influence the eating behaviour of adolescents?: editorial. The South African Journal of Clinical Nutrition, 23(2), 62-63. 
Story, M., Stang, J. (2005). Understanding adolescent eating behaviors. Guidelines for Adolescent Nutrition Services. Minneapolis: Centre for Leadership, Education and Training in Maternal and Child Nutrition Division of Epidemiology and Community Health. School of Public Health, University of Minnesota, p. 9-19.

TBSA (2010). Türkiye Beslenme ve Sağlık Araştırması. Saha Uygulaması El Kitabı. (p,139-144), T.C. Sağlık Bakanlığ 1 , Ankara. Code: 7561999 http://ekutuphane.sagem.gov.tr/kitaplar/turkiye beslenme ve saglik arastirmasi (tbsa) 2010 saha uygulamasi el kitabi.pdf (accessed 25.07.2018)
TUBER (2015). Turkiye beslenme rehberi. T.C. Saglik Bakanligi, Halk Sagligi Kurumu, Basim: Kayhan Ajans. Ankara 2016, ISBN : 978-975-590-608-9.

Willett W C and McCullough M L. (2008). Dietary pattern analysis for the evaluation of dietary guidelines. Asia Pacific Journal of Clinical Nutrition, 17(S1), 75-78.

Wordll, D., Daratha, K., Mandal, B., Bindler, R., Butkus, S.N. (2012). Changes in a middle school food environment affect food behavior and food choices. The Academy of Nutrition and Dietetics, 112, 137-41.

Wouters, E.J., Larsen, J.K., Kremers, S.P., Dagnelie, P.C., Geenen, R. (2010). Peer influence on snacking behavior in adolescence. Appetite, 55, 11-17. 